\title{
DIRECTIONAL MAXIMAL OPERATORS AND RADIAL WEIGHTS ON THE PLANE
}

\author{
HIROKI SAITO ${ }^{\otimes}$ and HITOSHI TANAKA
}

(Received 26 May 2013; accepted 17 July 2013; first published online 12 September 2013)

\section{Abstract}

Let $\Omega$ be the set of unit vectors and $w$ be a radial weight on the plane. We consider the weighted directional maximal operator defined by

$$
M_{\Omega, w} f(x):=\sup _{x \in R \in \mathcal{B}_{\Omega}} \frac{1}{w(R)} \int_{R}|f(y)| w(y) d y,
$$

where $\mathcal{B}_{\Omega}$ denotes the set of all rectangles on the plane whose longest side is parallel to some unit vector in $\Omega$ and $w(R)$ denotes $\int_{R} w$. In this paper we prove an almost-orthogonality principle for this maximal operator under certain conditions on the weight. The condition allows us to get the weighted norm inequality

$$
\left\|M_{\Omega, w} f\right\|_{L^{2}(w)} \leq C \log N\|f\|_{L^{2}(w)},
$$

when $w(x)=|x|^{a}, a>0$, and when $\Omega$ is the set of unit vectors on the plane with cardinality $N$ sufficiently large.

2010 Mathematics subject classification: primary 42B25.

Keywords and phrases: almost-orthogonality principle, directional maximal operator, radial weight, strong-type estimate.

\section{Introduction}

Fix a sufficiently large natural number $N$, denoted by $N \gg 1$. For a real number $a>0$, let $\mathcal{B}_{a, N}$ be the family of all cylinders in the $n$-dimensional Euclidean space $\mathbb{R}^{n}, n \geq 2$, which are congruent to the cylinders with height $N a$ and width $a$, but with arbitrary directions and centres. For a locally integrable function $f$ on $\mathbb{R}^{n}$ the 'small' Kakeya maximal operator $\mathcal{K}_{a, N}$ is defined by

$$
\mathcal{K}_{a, N} f(x):=\sup _{x \in R \in \mathcal{B}_{a, N}} \frac{1}{|R|} \int_{R}|f(y)| d y
$$

The second author is supported by the FMSP program at the Graduate School of Mathematical Sciences, University of Tokyo, Grant-in-Aid for Scientific Research (C) (No. 23540187), the Japan Society for the Promotion of Science, and was supported by the Fūjyukai foundation.

(C) 2013 Australian Mathematical Publishing Association Inc. 0004-9727/2013 \$16.00 
and the Kakeya maximal operator $\mathcal{K}_{N}$ is defined by

$$
\mathcal{K}_{N} f(x):=\sup _{a>0} \mathcal{K}_{a, N} f(x),
$$

where $|R|$ denotes the Lebesgue measure on $R$. It has been conjectured that $\mathcal{K}_{N}$ is bounded on $L^{n}\left(\mathbb{R}^{n}\right)$ with the norm growing no faster than $O\left((\log N)^{\alpha_{n}}\right)$ for some $\alpha_{n}>0$ as $N \rightarrow \infty$. In the case $n=2$, this conjecture was solved affirmatively by Córdoba [5] with the exponent $\alpha_{2}=2$ and re-proved by Strömberg [12] with $\alpha_{2}=1$. In the higher-dimensional case, $n>2$, these estimates have been proved so far only for some restricted class of functions. For functions of product type $f(x)=f_{1}\left(x_{1}\right) f_{2}\left(x_{2}\right) \cdots f_{n}\left(x_{n}\right)$, Igari [8] proved the estimate for $\mathcal{K}_{a, N}$ with the exponent $\alpha_{n}=3 / 2$ and the second author [13] re-proved this with the exponent $\alpha_{n}=(n-1) / n$. When the functions are of radial type $f(x)=f_{0}\left(\|x\|_{l^{2}}\right)$, Carbery et al. [4] proved the estimate for $\mathcal{K}_{N}$ with the exponent $\alpha_{n}=1$. In [14], for functions of radial type $f(x)=f_{0}\left(\|x\|_{l^{1}}\right)$, the second author proved the estimate for $\mathcal{K}_{a, N}$ with the exponent $\alpha_{n}=1$. In [6], for functions of radial type $f(x)=f_{0}\left(\|x\|_{l^{q}}\right), 1 \leq q \leq n$, Duoandikoetxea and Naibo proved the estimate for $\mathcal{K}_{N}$ with the exponent $\alpha_{n}=1$.

A more powerful but complicated maximal operator has been studied on the plane. Let $\Omega$ be a set of unit vectors in $\mathbb{R}^{2}$ with cardinality $N$. For a locally integrable function $f$ on $\mathbb{R}^{2}$, the directional maximal operator $M_{\Omega}$ is defined by

$$
M_{\Omega} f(x):=\sup _{r>0, \omega \in \Omega} \frac{1}{2 r} \int_{-r}^{r}|f(x+t \omega)| d t .
$$

Strömberg showed in [12] that if $\Omega$ is an equidistributed set of directions with cardinality $N$ then

$$
\left\|M_{\Omega} f\right\|_{L^{2}\left(\mathbb{R}^{2}\right)} \leq C \log N\|f\|_{L^{2}\left(\mathbb{R}^{2}\right)} .
$$

Notice that (1.1) yields the sharp $L^{2}\left(\mathbb{R}^{2}\right)$ estimate of the Kakeya maximal operator $\mathcal{K}_{N}$, since

$$
\mathcal{K}_{N} f(x) \leq C M_{\Omega} f(x) .
$$

In [9] and [10], Katz established that (1.1) holds without the condition that $\Omega$ is an equidistributed set of directions. In [4] and [6], for functions of radial type $f(x)=f_{0}\left(\|x\|_{l^{q}}\right), 1 \leq q \leq n$, it is essentially proved that

$$
\left\|M_{\Omega} f\right\|_{L^{n}\left(\mathbb{R}^{n}\right)} \leq C \log N\|f\|_{L^{n}\left(\mathbb{R}^{n}\right)} .
$$

In [1] and [2], Alfonseca et al. proposed a new method to study this operator and obtained a simple proof of the Katz result. In this paper we investigate the weighted version of their method and obtain a weighted version of the Katz result. To state our theorem, we first introduce some notation due to [1] and [2].

Let $\Omega$ be a subset of $[0, \pi / 4)$ and $w$ be a weight on $\mathbb{R}^{2}$. We define the weighted directional maximal operator $M_{\Omega, w}$, acting on locally integrable functions $f$ on $\mathbb{R}^{2}$, by

$$
M_{\Omega, w} f(x):=\sup _{x \in R \in \mathcal{B}_{\Omega}} \frac{1}{w(R)} \int_{R}|f(y)| w(y) d y,
$$


where $\mathcal{B}_{\Omega}$ denotes the basis of all rectangles with longest side forming an angle $\theta$ with the $x$-axis for some $\theta \in \Omega$, and $w(R)$ denotes $\int_{R} w$. Let $\Omega_{0}=\left\{\theta_{1}>\theta_{2}>\cdots>\theta_{j}>\cdots\right\}$ be an ordered subset of $\Omega$. We take $\theta_{0}=\pi / 4$ and consider, for each $j \geq 1$, sets $\Omega_{j}=\left[\theta_{j}, \theta_{j-1}\right) \cap \Omega$, such that $\theta_{j} \in \Omega_{0}$ for all $j$. Assume also that $\Omega=\bigcup \Omega_{j}$. To each set $\Omega_{j}, j=0,1,2, \ldots$, we associate the corresponding basis $\mathcal{B}_{j}$. We define the weighted maximal operators associated to each basis for $\Omega_{j}$ by

$$
M_{\Omega_{j}, w} f(x):=\sup _{x \in R \in \mathcal{B}_{j}} \frac{1}{w(R)} \int_{R}|f(y)| w(y) d y, \quad j=0,1,2, \ldots
$$

Throughout this paper we always assume that the weight $w$ is a radial weight: $w(x)=w_{0}\left(\|x\|_{l^{2}}\right)=w_{0}(|x|)$ for some nonnegative function $w_{0}$ on $\mathbb{R}_{+}$. We assume further that $w_{0}$ satisfies the following two conditions.

Doubling Condition. For all $0 \leq r_{1} \leq r_{1}^{\prime} \leq r_{2}^{\prime} \leq r_{2}<\infty$ with $r_{2}-r_{1}=2\left(r_{2}^{\prime}-r_{1}^{\prime}\right)$,

$$
\int_{r_{1}}^{r_{2}} w_{0}(r) d r \leq C \int_{r_{1}^{\prime}}^{r_{2}^{\prime}} w_{0}(r) d r .
$$

Supremum CONDITION. For all $0<r_{1}<r_{2}<\infty$,

$$
\sup _{r_{1}<r<r_{2}} w_{0}(r) \leq \frac{C}{r_{2}-r_{1}} \int_{r_{1}}^{r_{2}} w_{0}(r) d r .
$$

Notice that $r^{a}$ with $a>0$ satisfies these conditions. Indeed, the doubling condition is clear and, for all $0<r_{1}<r_{2}<\infty$,

$$
\left(r_{2}\right)^{a}=\frac{a+1}{r_{2}} \int_{0}^{r_{2}} r^{a} d r \leq \frac{a+1}{r_{2}-r_{1}} \int_{r_{1}}^{r_{2}} r^{a} d r .
$$

The main result of this paper is the following theorem.

THEOREM 1.1. Let $w$ be a radial weight satisfying (1.2) and (1.3). Then there exists a constant $C$ independent of $\Omega$ such that

$$
\left\|M_{\Omega, w}\right\|_{L^{2}(w) \rightarrow L^{2}(w)} \leq \sup _{j \geq 1}\left\|M_{\Omega_{j}, w}\right\|_{L^{2}(w) \rightarrow L^{2}(w)}+C\left\|M_{\Omega_{0}, w}\right\|_{L^{2}(w) \rightarrow L^{2}(w)},
$$

where $\|T\|_{L^{2}(w) \rightarrow L^{2}(w)}$ denotes the operator norm $T: L^{2}(w) \rightarrow L^{2}(w)$.

It is known that the weight $|x|^{a}, a>0$, is in $A_{\infty}^{*}\left(\mathbb{R}^{2}\right)$ (see [11, page 236]), where $A_{\infty}^{*}\left(\mathbb{R}^{2}\right)$ is the Muckenhoupt weight classes replacing the cubes $Q$ by the rectangles $R$ with sides parallel to the coordinate axes. From this fact and rotation invariance of the radial weights we can apply the proof of Corollary 4 in [2], and this allows us to give a weighted estimate of the Katz result (see [7, Theorems 6.7 and 6.13]).

Corollary 1.2. Let $\Omega$ be a set of unit vectors on $\mathbb{R}^{2}$ with cardinality $N \gg 1$ and $w(x)=|x|^{a}, a>0$. Then there exists a constant $C$ depending only on a such that

$$
\left\|M_{\Omega, w}\right\|_{L^{2}(w) \rightarrow L^{2}(w)} \leq C \log N .
$$


To prove Theorem 1.1, we essentially adapt the arguments in [1, 2]. In particular, the following is a weighted version of the key geometric observation used in [1].

Proposition 1.3. Let $0<\theta_{1}, \theta_{2}<\pi / 4$. Let

$$
\omega_{0}=(1,0), \quad \omega_{1}=\left(\cos \theta_{1}, \sin \theta_{1}\right) \quad \text { and } \quad \omega_{2}=\left(\cos \left(-\theta_{2}\right), \sin \left(-\theta_{2}\right)\right) .
$$

Let $B$ be a rectangle whose longest side is parallel to $\omega_{1}$ and let $R$ be a rectangle whose longest side is parallel to $\omega_{2}$. Suppose that $B \cap R \neq \emptyset$ and that the long side length of $B$ is bigger than that of $R$. Then there exists a rectangle $\widetilde{R} \supset R$ whose longest side is parallel to $\omega_{0}$ such that

$$
\frac{w(R \cap B)}{w(R)} \leq C \frac{w(\widetilde{R} \cap B)}{w(\widetilde{R})},
$$

where the constant $C$ is independent of $\theta_{1}, \theta_{2}, B$ and $R$.

To prove the proposition we need several technical lemmas.

We briefly describe the rest of this paper. In Section 2 we show Proposition 1.3. Several technical lemmas are also shown in this section. By using Proposition 1.3, we show Theorem 1.1 in Section 3.

\section{Geometry on the plane}

The aim of this section is to prove Proposition 1.3. To do so we first introduce our notation. We write $X \lesssim Y, Y \gtrsim X$ if there is a constant $C$ such that $X \leq C Y$. The constant $C$ may vary from line to line but the constants with subscripts, such as $C_{1}$, $C_{2}$, do not change in different occurrences. We write $X \approx Y$ if $X \lesssim Y$ and $X \gtrsim Y$.

Given rectangle $R \subset \mathbb{R}^{2}$, let $c R$ be the rectangle with the same centre as $R$, but with sides $c$ times as long. Given a measurable set $E \subset \mathbb{R}^{2}$, let $|E|$ denote the Lebesgue measure of $E$ and $w(E)$ denote $\int_{E} w$.

Our first task is to show two key lemmas.

2.1. First key lemma. Recall that we always suppose that the weight $w$ satisfies $w(x)=w_{0}(|x|)$ and that $w_{0}$ satisfies the doubling condition (1.2) and the supremum condition (1.3). For an $A \subset \mathbb{R}^{2}$ we set $r_{1}(A):=\inf _{x \in A}|x|, r_{2}(A):=\sup _{x \in A}|x|$ and $\operatorname{rad}(A):=r_{2}(A)-r_{1}(A)$. By definition we can easily see that, if $A \subset B \subset \mathbb{R}^{2}$, then $\operatorname{rad}(A) \leq \operatorname{rad}(B)$. We also see that $\operatorname{rad}(2 R) \lesssim \operatorname{rad}(R)$ for any rectangle $R \subset \mathbb{R}^{2}$. The following is our first key lemma.

Lemma 2.1. Let $R \subset \mathbb{R}^{2}$ be a rectangle. Then

$$
\frac{w(R)}{|R|} \approx \frac{1}{\operatorname{rad}(R)} \int_{r_{1}(R)}^{r_{2}(R)} w_{0}(r) d r .
$$

Proof. Notice that

$$
w(R)=\int_{r_{1}(R)}^{r_{2}(R)} \operatorname{arc}\left(R \cap C_{r}\right) w_{0}(r) d r
$$


where $C_{r}$ is the circle of radius $r$ and centred at the origin and $\operatorname{arc}\left(R \cap C_{r}\right)$ is the arc length of the arc $R \cap C_{r}$. It follows from (2.1) and the supremum condition (1.3) that

$$
\begin{aligned}
\frac{w(R)}{|R|} & =\frac{1}{|R|} \int_{r_{1}(R)}^{r_{2}(R)} \operatorname{arc}\left(R \cap C_{r}\right) w_{0}(r) d r \\
& \leq \sup _{r_{1}(R)<r<r_{2}(R)} w_{0}(r) \cdot \frac{1}{|R|} \int_{r_{1}(R)}^{r_{2}(R)} \operatorname{arc}\left(R \cap C_{r}\right) d r \\
& =\sup _{r_{1}(R)<r<r_{2}(R)} w_{0}(r) \lesssim \frac{1}{\operatorname{rad}(R)} \int_{r_{1}(R)}^{r_{2}(R)} w_{0}(r) d r .
\end{aligned}
$$

Thus, we shall prove the converse,

$$
\frac{w(R)}{|R|} \gtrsim \frac{1}{\operatorname{rad}(R)} \int_{r_{1}(R)}^{r_{2}(R)} w_{0}(r) d r .
$$

Since $w_{0}$ satisfies the doubling condition (1.2), we need only verify the following claim.

Claim. There exists a set $A \subset R$ such that

$$
\operatorname{rad}(R) \leq C_{1} \operatorname{rad}(A)
$$

and

$$
\operatorname{rad}(A) \inf _{r_{1}(A)<r<r_{2}(A)} \operatorname{arc}\left(A \cap C_{r}\right) \geq C_{2}|R|,
$$

where the constants $C_{1}$ and $C_{2}$ are independent of $R$ and $A$.

If this claim is true, then it follows from (2.1) and the doubling condition (1.2) that

$$
\begin{aligned}
w(R) & \geq \int_{r_{1}(A)}^{r_{2}(A)} w_{0}(r) d r \cdot \inf _{r_{1}(A)<r<r_{2}(A)} \operatorname{arc}\left(A \cap C_{r}\right) \\
& \gtrsim \frac{1}{\operatorname{rad}(R)} \int_{r_{1}(R)}^{r_{2}(R)} w_{0}(r) d r \cdot \operatorname{rad}(A) \inf _{r_{1}(A)<r<r_{2}(A)} \operatorname{arc}\left(A \cap C_{r}\right) \\
& \gtrsim \frac{1}{\operatorname{rad}(R)} \int_{r_{1}(R)}^{r_{2}(R)} w_{0}(r) d r \cdot|R| .
\end{aligned}
$$

We now prove the claim.

Because of the rotation invariance and the symmetry of the problem, we may assume that the rectangle $R$ forms

$$
R=\left(a_{1}, a_{2}\right) \times\left(b_{1}, b_{2}\right), \quad 0<a_{1}<a_{2}<\infty, \quad 0<b_{1}<b_{2}<a_{2} .
$$

Let

$$
r_{1}=\sqrt{a_{1}^{2}+b_{1}^{2}}, \quad r_{2}=\sqrt{a_{2}^{2}+b_{1}^{2}}, \quad r_{3}=\sqrt{a_{1}^{2}+b_{2}^{2}} \quad \text { and } \quad r_{4}=\sqrt{a_{2}^{2}+b_{2}^{2}} .
$$

Then $r_{1}=r_{1}(R)$ and $r_{4}=r_{2}(R)$ and a simple calculation shows that

$$
r_{3}-r_{1} \geq r_{4}-r_{2} \quad \text { and } \quad r_{2}-r_{1} \geq r_{4}-r_{3} .
$$

We now consider two cases. 
THE CASE $r_{2} \leq r_{3}$. For $t \geq-1$, we set

$$
u(t):=\sqrt{a_{2}^{2}+t\left(a_{2}^{2}-a_{1}^{2}\right)}
$$

Let

$$
t_{1}=\frac{b_{1}^{2}}{a_{2}^{2}-a_{1}^{2}} \quad \text { and } \quad t_{2}=\frac{b_{2}^{2}}{a_{2}^{2}-a_{1}^{2}}-1
$$

Then we observe that

$$
r_{1}=u\left(t_{1}-1\right), \quad r_{2}=u\left(t_{1}\right), \quad r_{3}=u\left(t_{2}\right), \quad r_{4}=u\left(t_{2}+1\right),
$$

and hence $t_{1} \leq t_{2}$. We choose an $A \subset R$ to be a set lying between the circles $C_{u\left(t_{1}-1 / 2\right)}$ and $C_{r_{3}}$.

We first show (2.2). It follows that

$$
\begin{aligned}
\frac{r_{2}-r_{1}}{r_{2}-u\left(t_{1}-1 / 2\right)} & =\frac{u\left(t_{1}\right)-u\left(t_{1}-1\right)}{u\left(t_{1}\right)-u\left(t_{1}-1 / 2\right)}=2 \frac{u\left(t_{1}\right)+u\left(t_{1}-1 / 2\right)}{u\left(t_{1}\right)+u\left(t_{1}-1\right)} \\
& \leq 2 \frac{2 u\left(t_{1}\right)}{u\left(t_{1}\right)}=4 .
\end{aligned}
$$

This and (2.4) imply

$$
\begin{aligned}
r_{4}-r_{1} & =\left(r_{4}-r_{3}\right)+\left(r_{3}-r_{2}\right)+\left(r_{2}-r_{1}\right) \\
& \leq\left(r_{3}-r_{2}\right)+2\left(r_{2}-r_{1}\right) \\
& \leq 8\left(r_{3}-r_{2}\right)+8\left(r_{2}-u\left(t_{1}-1 / 2\right)\right) \\
& =8\left(r_{3}-u\left(t_{1}-1 / 2\right)\right),
\end{aligned}
$$

which means $\operatorname{rad}(R) \leq 8 \operatorname{rad}(A)$ and proves (2.2).

We next show (2.3). Observe that if $t \in\left[t_{1}, t_{2}\right]$ then the circle $C_{u(t)}$ intersects with both vertical sides of $R$. Furthermore, we observe that the circle $C_{u(t)}$ intersects with the vertical line $x=a_{2}$ at the height $\sqrt{t} \sqrt{a_{2}^{2}-a_{1}^{2}}$ and intersects with the vertical line $x=a_{1}$ at the height $\sqrt{t+1} \sqrt{a_{2}^{2}-a_{1}^{2}}$ (see Figure 1). Hence, for all $t_{1} \leq t \leq t_{2}$,

$$
\operatorname{arc}\left(R \cap C_{u(t)}\right) \geq(\sqrt{t+1}-\sqrt{t}) \sqrt{a_{2}^{2}-a_{1}^{2}} \geq \frac{\sqrt{a_{2}^{2}-a_{1}^{2}}}{2 \sqrt{t+1}}
$$

Thus

$$
\inf _{r_{2}<r<r_{3}} \operatorname{arc}\left(A \cap C_{r}\right) \geq \frac{\sqrt{a_{2}^{2}-a_{1}^{2}}}{2 \sqrt{t_{2}+1}} \geq \frac{\sqrt{a_{2}^{2}-a_{1}^{2}}}{4 \sqrt{t_{2}+1}} .
$$

We also observe that the circle $C_{u\left(t_{1}-1 / 2\right)}$ intersects with the vertical line $x=a_{1}$ at the height

$$
\sqrt{t_{1}+1 / 2} \sqrt{a_{2}^{2}-a_{1}^{2}}
$$




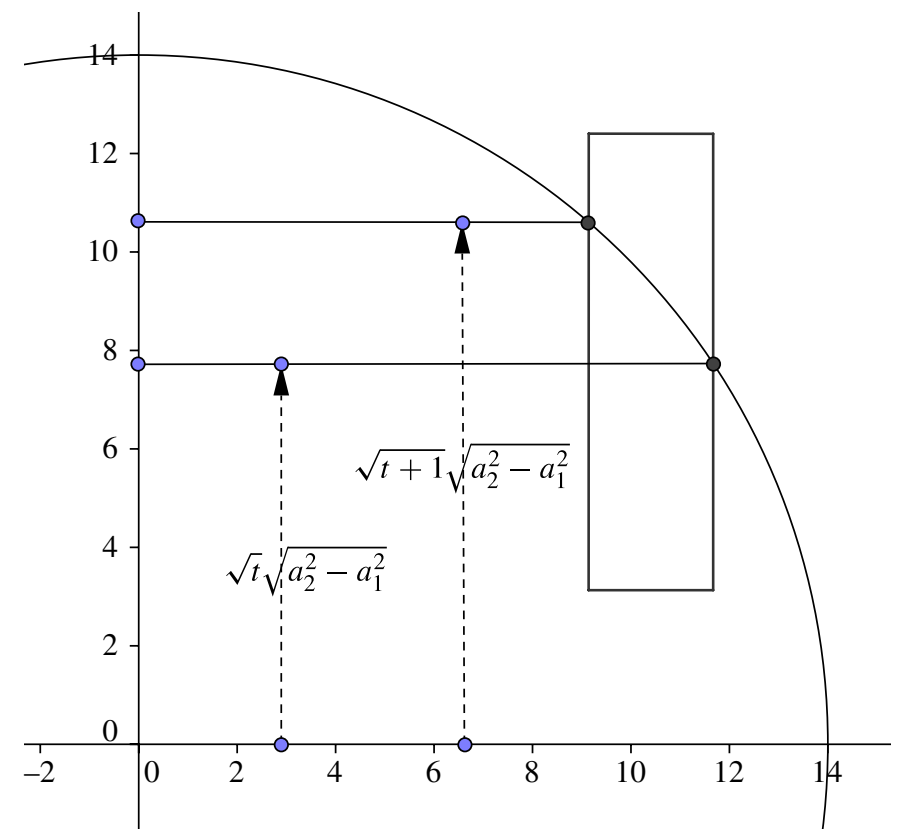

Figure 1. The circle $C_{u(t)}$ intersects with both vertical sides of $R$.

Then

$$
\begin{aligned}
\inf _{u\left(t_{1}-1 / 2\right)<r<r_{2}} \operatorname{arc}\left(A \cap C_{r}\right) & =\operatorname{arc}\left(R \cap C_{u\left(t_{1}-1 / 2\right)}\right) \\
& \geq\left(\sqrt{t_{1}+1 / 2}-\sqrt{t_{1}}\right) \sqrt{a_{2}^{2}-a_{1}^{2}} \\
& \geq \frac{\sqrt{a_{2}^{2}-a_{1}^{2}}}{4 \sqrt{t_{1}+1 / 2}} .
\end{aligned}
$$

Thus, by (2.5) and $t_{1} \leq t_{2}$,

$$
\begin{aligned}
\left(r_{3}-\right. & \left.u\left(t_{1}-1 / 2\right)\right) \inf _{u\left(t_{1}-1 / 2\right)<r<r_{3}} \operatorname{arc}\left(R \cap C_{r}\right) \\
& \geq\left(r_{3}-u\left(t_{1}-1 / 2\right)\right) \frac{\sqrt{a_{2}^{2}-a_{1}^{2}}}{4 \sqrt{t_{2}+1}} \\
& =\frac{1}{r_{3}+u\left(t_{1}-1 / 2\right)} \frac{1 / 2+t_{2}-t_{1}}{4 \sqrt{t_{2}+1}} \sqrt{a_{2}^{2}-a_{1}^{2}}\left(a_{2}-a_{1}\right)\left(a_{2}+a_{1}\right) \\
& \geq \frac{a_{2}+a_{1}}{8\left(r_{3}+u\left(t_{1}-1 / 2\right)\right)}\left(\sqrt{t_{2}+1}-\sqrt{t_{1}}\right) \sqrt{a_{2}^{2}-a_{1}^{2}}\left(a_{2}-a_{1}\right) \\
& \geq \frac{a_{2}}{32 a_{2}}|R|=\frac{|R|}{32}
\end{aligned}
$$


where we have used

$$
\begin{aligned}
\frac{1 / 2+t_{2}-t_{1}}{4 \sqrt{t_{2}+1}} & =\frac{1+2\left(t_{2}-t_{1}\right)}{8 \sqrt{t_{2}+1}} \geq \frac{t_{2}+1-t_{1}}{8 \sqrt{t_{2}+1}} \\
& =\frac{1}{8}\left(\sqrt{t_{2}+1}-\frac{t_{1}}{\sqrt{t_{2}+1}}\right) \geq \frac{1}{8}\left(\sqrt{t_{2}+1}-\sqrt{t_{1}}\right)
\end{aligned}
$$

and

$$
\left(\sqrt{t_{2}+1}-\sqrt{t_{1}}\right) \sqrt{a_{2}^{2}-a_{1}^{2}}\left(a_{2}-a_{1}\right)=\left(b_{2}-b_{1}\right)\left(a_{2}-a_{1}\right)=|R| .
$$

These prove (2.3) in this case.

The CASE $r_{2}>r_{3}$. For $t \geq-1$, we set

$$
v(t):=\sqrt{b_{2}^{2}+t\left(b_{2}^{2}-b_{1}^{2}\right)} .
$$

Let

$$
t_{3}=\frac{a_{1}^{2}}{b_{2}^{2}-b_{1}^{2}} \quad \text { and } \quad t_{4}=\frac{a_{2}^{2}}{b_{2}^{2}-b_{1}^{2}}-1
$$

Then

$$
r_{1}=v\left(t_{3}-1\right), \quad r_{3}=v\left(t_{3}\right), \quad r_{2}=v\left(t_{4}\right), \quad r_{4}=v\left(t_{4}+1\right),
$$

and hence $t_{3} \leq t_{4}$. We choose an $A \subset R$ to be a set lying between the circles $C_{v\left(t_{3}-1 / 2\right)}$ and $C_{r_{2}}$.

As in the previous case, we start by showing (2.2). It follows that

$$
\frac{r_{3}-r_{1}}{r_{3}-v\left(t_{3}-1 / 2\right)}=\frac{v\left(t_{3}\right)-v\left(t_{3}-1\right)}{v\left(t_{3}\right)-v\left(t_{3}-1 / 2\right)} \leq 4
$$

This and (2.4) imply

$$
\begin{aligned}
r_{4}-r_{1} & =\left(r_{4}-r_{2}\right)+\left(r_{2}-r_{3}\right)+\left(r_{3}-r_{1}\right) \\
& \leq\left(r_{2}-r_{3}\right)+2\left(r_{3}-r_{1}\right) \\
& \leq 8\left(r_{2}-r_{3}\right)+8\left(r_{3}-v\left(t_{3}-1 / 2\right)\right) \\
& =8\left(r_{2}-v\left(t_{3}-1 / 2\right)\right),
\end{aligned}
$$

which means $\operatorname{rad}(R) \leq 8 \operatorname{rad}(A)$ and proves (2.2).

We next show (2.3). Observe that

$$
\inf _{r_{3}<r<r_{2}} \operatorname{arc}\left(A \cap C_{r}\right) \geq b_{2}-b_{1} .
$$

We also observe that the circle $C_{v\left(t_{3}-1 / 2\right)}$ intersects with the vertical line $x=a_{1}$ at the height

$$
\sqrt{\left(b_{1}^{2}+b_{2}^{2}\right) / 2}
$$


which gives that

$$
\begin{aligned}
\inf _{v\left(t_{3}-1 / 2\right)<r<r_{3}} \operatorname{arc}\left(A \cap C_{r}\right) & \geq \sqrt{\left(b_{1}^{2}+b_{2}^{2}\right) / 2}-b_{1} \geq \frac{\left(b_{2}^{2}-b_{1}^{2}\right) / 2}{\sqrt{\left(b_{1}^{2}+b_{2}^{2}\right) / 2}+b_{1}} \\
& \geq \frac{b_{2}+b_{1}}{4 b_{2}}\left(b_{2}-b_{1}\right) \geq \frac{b_{2}-b_{1}}{4},
\end{aligned}
$$

where we have used $b_{2}>b_{1}$. Notice that

$$
\begin{aligned}
r_{4}-r_{1} & =\sqrt{a_{2}^{2}+b_{2}^{2}}-\sqrt{a_{1}^{2}+b_{1}^{2}}=\frac{a_{2}^{2}+b^{2}-a_{1}^{2}-b_{1}^{2}}{\sqrt{a_{2}^{2}+b_{2}^{2}}+\sqrt{a_{1}^{2}+b_{1}^{2}}} \\
& \geq \frac{\left(a_{2}-a_{1}\right)\left(a_{2}+a_{1}\right)}{2 \sqrt{2} a_{2}} \geq \frac{a_{2}-a_{1}}{2 \sqrt{2}},
\end{aligned}
$$

where we have used $a_{2}>b_{2}>b_{1}>0$ and $a_{2}>a_{1}$. Thus,

$$
\begin{aligned}
& \left(r_{3}-v\left(t_{3}-1 / 2\right)\right) \inf _{v\left(t_{3}-1 / 2\right)<r<r_{3}} \operatorname{arc}\left(R \cap C_{r}\right) \\
& \quad \geq \frac{1}{32}\left(r_{4}-r_{1}\right)\left(b_{2}-b_{1}\right) \geq \frac{\sqrt{2}}{128}\left(a_{2}-a_{1}\right)\left(b_{2}-b_{1}\right)=\frac{\sqrt{2}}{128}|R|,
\end{aligned}
$$

which proves (2.3) in this case, and, the proof of Lemma 2.1 is now complete.

2.2. Second key lemma. We next show the second key lemma.

LEMMA 2.2. Let $R$ be a rectangle which lies on the upper half plane and whose sides are parallel to the $x$-and y-axes with height $2 n$ and width $2 m, m>n>0$. Let $\mathrm{C}_{0}=(a, b)$ be the centre of $R$. Set

$$
\begin{aligned}
\mathrm{A}_{0}=(a, b)+(-m, n), & \mathrm{A}_{1}=(a, b)+(m, n), \\
\mathrm{B}_{0}=(a, b)+(-m,-n), & \mathrm{B}_{1}=(a, b)+(m,-n) .
\end{aligned}
$$

Then there exists a constant $C>0$ such that the following statements hold.

(a) When $a \leq m$ and $b>n$,

$$
\frac{\operatorname{rad}(R)}{\operatorname{rad}\left(\mathrm{A}_{0} \mathrm{~B}_{1}\right)} \leq C \text {. }
$$

(b) When $a>m$ and $b>n$,

$$
\min \left\{\frac{\operatorname{rad}(R)}{\operatorname{rad}\left(\mathrm{A}_{0} \mathrm{~B}_{1}\right)}, \frac{\operatorname{rad}(R)}{\operatorname{rad}\left(\mathrm{B}_{0} \mathrm{~B}_{1}\right)}\right\} \leq C
$$

and

$$
\min \left\{\frac{\operatorname{rad}(R)}{\operatorname{rad}\left(\mathrm{A}_{0} \mathrm{~B}_{1}\right)}, \frac{\operatorname{rad}(R)}{\operatorname{rad}\left(\mathrm{A}_{0} \mathrm{~B}_{0}\right)}\right\} \leq C .
$$


Proof. Let $\mathrm{D}$ be the point on the line joining $\mathrm{A}_{0}$ and $\mathrm{B}_{1}$ which is closest to the origin. Then $\mathrm{D}$ lies on the line $l:-m x+n y=0$. We let $\mathrm{D}_{0} \in \mathrm{A}_{0} \mathrm{~B}_{1}$ be the closest point from the origin to the line segment $\mathrm{A}_{0} \mathrm{~B}_{1}$ and let $\mathrm{D}_{1} \in R$ be the closest point from the origin to the rectangle $R$. By the definition we have $r_{1}\left(\mathrm{~A}_{0} \mathrm{~B}_{1}\right)=\left\|\mathrm{D}_{0}\right\|, r_{1}(R)=\left\|\mathrm{D}_{1}\right\|$ and $\|\mathrm{D}\| \leq\left\|\mathrm{D}_{0}\right\|$.

Proof of (a). It is clear that if $R$ lies on the second quadrant, then $\operatorname{rad}(R)=\operatorname{rad}\left(\mathrm{A}_{0} \mathrm{~B}_{1}\right)$. So, we prove the statement in three cases:

(i) $\quad-m \leq a \leq 0$ and $b>n$;

(ii) $m \geq a>0, b>n$ and $\mathrm{C}_{0}$ lies above the line $l$;

(iii) $m \geq a>0, b>n$ and $\mathrm{C}_{0}$ lies below the line $l$.

Case (i). If $-m \leq a \leq 0$ and $b>n$, then $r_{2}(R)=r_{2}\left(\mathrm{~A}_{0} \mathrm{~B}_{1}\right)=\left\|\mathrm{A}_{0}\right\|, \mathrm{D}_{1}=(0, b-n)$ and $\mathrm{C}_{0}$ lies above the line $l$. Thus, $-m a+n b>0$ and

$$
\operatorname{rad}\left(\mathrm{A}_{0} \mathrm{~B}_{1}\right)=\left\|\mathrm{A}_{0}\right\|-\left\|\mathrm{D}_{0}\right\| \geq\left\|\mathrm{A}_{0}\right\|-\left\|\mathrm{C}_{0}\right\| .
$$

Hence,

$$
\begin{aligned}
\frac{\operatorname{rad}(R)}{\operatorname{rad}\left(\mathrm{A}_{0} \mathrm{~B}_{1}\right)} & \leq \frac{\left\|\mathrm{A}_{0}\right\|-\left\|\mathrm{D}_{1}\right\|}{\left\|\mathrm{A}_{0}\right\|-\left\|\mathrm{C}_{0}\right\|}=\frac{\left\|\mathrm{A}_{0}\right\|^{2}-\left\|\mathrm{D}_{1}\right\|^{2}}{\left\|\mathrm{~A}_{0}\right\|^{2}-\left\|\mathrm{C}_{0}\right\|^{2}} \cdot \frac{\left\|\mathrm{A}_{0}\right\|+\left\|\mathrm{C}_{0}\right\|}{\left\|\mathrm{A}_{0}\right\|+\left\|\mathrm{D}_{1}\right\|} \leq 2 \frac{\left\|\mathrm{A}_{0}\right\|^{2}-\left\|\mathrm{D}_{1}\right\|^{2}}{\left\|\mathrm{~A}_{0}\right\|^{2}-\left\|\mathrm{C}_{0}\right\|^{2}} \\
& \lesssim \frac{a^{2}+m^{2}-2 m a+4 n b}{m^{2}+n^{2}+2(-m a+n b)} \lesssim \frac{2 m^{2}+4(-m a+n b)}{m^{2}+n^{2}+2(-m a+n b)} \lesssim 1,
\end{aligned}
$$

where we have used $-m a>0$ and $a^{2} \leq m^{2}$.

Case (ii). If $m \geq a>0, b>n$ and if $\mathrm{C}_{0}$ lies above the line $l$, then $r_{2}(R)=\left\|\mathrm{A}_{1}\right\|$, $\mathrm{D}_{1}=(0, b-n)$ and we have $-m a+n b>0$ and (2.6). Therefore,

$$
\frac{\operatorname{rad}(R)}{\operatorname{rad}\left(\mathrm{A}_{0} \mathrm{~B}_{1}\right)} \lesssim \frac{a^{2}+m^{2}+2 m a+4 n b}{m^{2}+n^{2}+2(-m a+n b)} \lesssim 1+\frac{n b}{m^{2}+n^{2}-m a+n b},
$$

where we have used $a \leq m$. Since $a \leq m, m^{2}+n^{2}-m a+n b \geq n^{2}+n b \geq n b$, and hence

$$
\frac{n b}{m^{2}+n^{2}-m a+n b} \leq 1 \text {. }
$$

Case (iii). If $m \geq a>0, b>n$ and if $\mathrm{C}_{0}$ lies below the line $l$, then $r_{2}(R)=\left\|\mathrm{A}_{1}\right\|$, $\mathrm{D}_{1}=(0, b-n)$ and we have $-m a+n b \leq 0$ and

$$
\operatorname{rad}\left(\mathrm{A}_{0} \mathrm{~B}_{1}\right)=\left\|\mathrm{B}_{1}\right\|-\left\|\mathrm{D}_{0}\right\| \geq\left\|\mathrm{B}_{1}\right\|-\left\|\mathrm{C}_{0}\right\| .
$$

Hence,

$$
\frac{\operatorname{rad}(R)}{\operatorname{rad}\left(\mathrm{A}_{0} \mathrm{~B}_{1}\right)} \lesssim \frac{a^{2}+m^{2}+2 m a+4 n b}{m^{2}+n^{2}+2(m a-n b)} \lesssim 1,
$$

where we have used $m^{2} \geq m a \geq n b$.

Proof of (b). As for part (a), we consider the following two cases:

(i) $\quad a>m, b>n$ and $\mathrm{C}_{0}$ lies above the line $l$;

(ii) $a>m, b>n$ and $\mathrm{C}_{0}$ lies below the line $l$. 


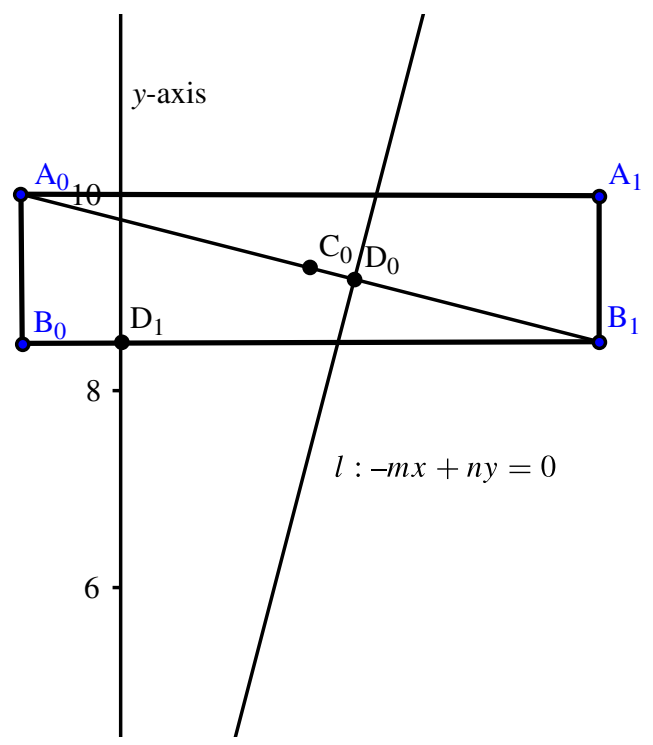

FIGURE 2. Proof of (a) case (ii).

Case (i). If $a>m, b>n$ and if $\mathrm{C}_{0}$ lies above the line $l$, then $-m a+n b>0$ and

$$
\operatorname{rad}(R)=\left\|\mathrm{A}_{1}\right\|-\left\|\mathrm{B}_{0}\right\|
$$

It then follows that

$$
\frac{\operatorname{rad}(R)}{\operatorname{rad}\left(\mathrm{A}_{0} \mathrm{~B}_{0}\right)} \lesssim \frac{4(m a+n b)}{4 n b} \lesssim 1,
$$

where we have used $n b>m a$. This implies that the second inequality of (b) holds.

We show the first inequality of (b). We recall that $-m a+n b>0$ and that (2.6) and (2.8) hold. Thus,

$$
\frac{\operatorname{rad}(R)}{\operatorname{rad}\left(\mathrm{A}_{0} \mathrm{~B}_{1}\right)} \lesssim \frac{4(m a+n b)}{m^{2}+n^{2}+2(-m a+n b)} \lesssim \frac{m a+n b}{-m a+n b}
$$

and

$$
\frac{\operatorname{rad}(R)}{\operatorname{rad}\left(\mathrm{B}_{0} \mathrm{~B}_{1}\right)} \lesssim \frac{m a+n b}{m a} .
$$

Now, under the condition $-m a+n b>0$, we shall estimate $\sup \min \{X, Y\}$, where

$$
X:=\frac{m a+n b}{-m a+n b} \quad \text { and } \quad Y:=\frac{m a+n b}{m a} .
$$

Set

$$
\left\{\begin{array}{lll}
\mathrm{C}_{0}=(a, b), & \mathrm{C}_{1}=(m, 0), & \mathrm{C}_{2}=(m, n), \\
\mathrm{C}_{3}=(n, m), & \mathrm{C}_{4}=(-m, n), & \mathrm{O}=(0,0)
\end{array}\right.
$$


Since

$$
\begin{gathered}
m a+n b=\left\|\mathrm{C}_{0}\right\|\left\|\mathrm{C}_{2}\right\| \cos \angle \mathrm{C}_{0} \mathrm{OC}_{2}, \\
-m a+n b=\left\|\mathrm{C}_{0}\right\|\left\|\mathrm{C}_{4}\right\| \cos \angle \mathrm{C}_{0} \mathrm{OC}_{4}, \\
m a=\left\|\mathrm{C}_{0}\right\|\left\|\mathrm{C}_{1}\right\| \cos \angle \mathrm{C}_{0} \mathrm{OC}_{1},
\end{gathered}
$$

we have

$$
X=\frac{m a+n b}{-m a+n b}=\frac{\cos \angle \mathrm{C}_{0} \mathrm{OC}_{2}}{\cos \angle \mathrm{C}_{0} \mathrm{OC}_{4}}, \quad Y=\frac{m a+n b}{m a} \leq \sqrt{2} \frac{\cos \angle \mathrm{C}_{0} \mathrm{OC}_{2}}{\cos \angle \mathrm{C}_{0} \mathrm{OC}_{1}},
$$

where the inequality $\sqrt{2} m=\sqrt{2 m^{2}} \geq \sqrt{m^{2}+n^{2}}$ is used. Moreover, as $\mathrm{C}_{0}$ is assumed to lie above the line $l$,

$$
\cos \angle \mathrm{C}_{0} \mathrm{OC}_{2} \leq \cos \angle \mathrm{C}_{3} \mathrm{OC}_{2}=\frac{2 m n}{m^{2}+n^{2}} .
$$

As

$$
\min \left\{\frac{1}{\cos \angle \mathrm{C}_{0} \mathrm{OC}_{4}}, \frac{1}{\cos \angle \mathrm{C}_{0} \mathrm{OC}_{1}}\right\}
$$

attains its maximum at $\angle \mathrm{C}_{0} \mathrm{OC}_{4}=\angle \mathrm{C}_{0} \mathrm{OC}_{1}$, it follows that

$$
\cos \angle \mathrm{C}_{0} \mathrm{OC}_{1}=\cos \left(\frac{\pi}{2}-\frac{\angle \mathrm{C}_{1} \mathrm{OC}_{2}}{2}\right)=\sin \frac{\angle \mathrm{C}_{1} \mathrm{OC}_{2}}{2} .
$$

Thus,

$$
\sup \min \left\{\frac{1}{\cos \angle \mathrm{C}_{0} \mathrm{OC}_{4}}, \frac{1}{\cos \angle \mathrm{C}_{0} \mathrm{OC}_{1}}\right\}=\frac{1}{\sin \frac{\angle \mathrm{C}_{1} \mathrm{OC}_{2}}{2}} \approx \frac{\sqrt{m^{2}+n^{2}}}{n} .
$$

In conclusion,

$$
\min \{X, Y\} \lesssim \frac{m n /\left(m^{2}+n^{2}\right)}{n / \sqrt{m^{2}+n^{2}}} \approx \frac{m}{\sqrt{m^{2}+n^{2}}} \lesssim 1 .
$$

Case (ii). If $a>m, b>n$ and if $\mathrm{C}_{0}$ lies below the line $l$, then $-m a+n b \leq 0$ and (2.8) holds. Thus, as $m a \geq n b$,

$$
\frac{\operatorname{rad}(R)}{\operatorname{rad}\left(\mathrm{B}_{0} \mathrm{~B}_{1}\right)} \lesssim \frac{4(m a+n b)}{4 m a} \lesssim 1 .
$$

The first inequality of (b) follows.

As in the previous case, we now show the second inequality of (b). The arguments are essentially the same as for case (i). First observe that since $-m a+n b \leq 0$, and since (2.7) and (2.8) hold,

$$
\frac{\operatorname{rad}(R)}{\operatorname{rad}\left(\mathrm{A}_{0} \mathrm{~B}_{1}\right)} \lesssim \frac{4(m a+n b)}{m^{2}+n^{2}+2(m a-n b)} \lesssim \frac{m a+n b}{m a-n b}
$$


and

$$
\frac{\operatorname{rad}(R)}{\operatorname{rad}\left(\mathrm{A}_{0} \mathrm{~B}_{0}\right)} \lesssim \frac{m a+n b}{n b} .
$$

Now, under the condition $-m a+n b \leq 0$, we shall estimate $\sup \min \left\{X^{\prime}, Y^{\prime}\right\}$, where

$$
X^{\prime}:=\frac{m a+n b}{m a-n b} \quad \text { and } \quad Y^{\prime}:=\frac{m a+n b}{n b}
$$

As observed before,

$$
\min \left\{X^{\prime}, Y^{\prime}\right\}=\min \left\{\frac{\cos \angle \mathrm{C}_{0} \mathrm{OC}_{2}}{\cos \angle \mathrm{C}_{0} \mathrm{OC}_{4}^{\prime}}, \frac{\sqrt{m^{2}+n^{2}}}{\sqrt{n^{2}}} \cdot \frac{\cos \angle \mathrm{C}_{0} \mathrm{OC}_{2}}{\cos \angle \mathrm{C}_{0} \mathrm{OC}_{1}^{\prime}}\right\},
$$

where $\mathrm{C}_{4}^{\prime}=(m,-n)$ and $\mathrm{C}_{1}^{\prime}=(0, n)$. Hence, $\sup \min \left\{X^{\prime}, Y^{\prime}\right\}$ is attained when

$$
\cos \angle \mathrm{C}_{0} \mathrm{OC}_{4}^{\prime}=\cos \angle \mathrm{C}_{0} \mathrm{OC}_{1}^{\prime}=\cos \left(\frac{\pi}{2}+\frac{\theta}{2}\right),
$$

where $\theta$ is the angle that the vector $(m, n)$ forms with the $x$-axis. Since $\theta / 2 \leq \pi / 8$, $\cos (\pi / 2+\theta / 2)$ is bounded from below and hence

$$
\min \left\{\frac{\cos \angle \mathrm{C}_{0} \mathrm{OC}_{2}}{\cos \angle \mathrm{C}_{0} \mathrm{OC}_{4}^{\prime}}, \frac{\sqrt{m^{2}+n^{2}}}{\sqrt{n^{2}}} \cdot \frac{\cos \angle \mathrm{C}_{0} \mathrm{OC}_{2}}{\cos \angle \mathrm{C}_{0} \mathrm{OC}_{1}^{\prime}}\right\} \leq \min \left\{C, \frac{\sqrt{m^{2}+n^{2}}}{\sqrt{n^{2}}} \cdot C\right\} \lesssim 1 .
$$

The proof of Lemma 2.2 is now complete.

2.3. Proof of Proposition 1.3. We use the formula proved in Lemma 2.1. Notice that

$$
w(R) \leq w(\hat{R}) \lesssim w(R) \quad \text { for any rectangle } R,
$$

where $\hat{R}$ is a rectangle with the same centre and the same short side length as $R$ but twice the long side length, or a rectangle with the same centre and the same long side length as $R$ but twice the short side length.

We now take rectangles $R^{\prime}$ and $B^{\prime}$ that satisfy the following conditions:

- $\quad R^{\prime}$ and $B^{\prime}$ have the common centre;

- $\quad R^{\prime}$ (respectively, $B^{\prime}$ ) is expanded from $R$ (respectively, $B$ ) toward the long sides;

- $\quad$ the long side of $R^{\prime}$ (respectively, $B^{\prime}$ ) is three times the length of that of $R$ (respectively, $B$ );

- $\quad R \cap B \subset R^{\prime} \cap B^{\prime}$.

Let $\widetilde{R^{\prime}}$ be a smallest rectangle in the direction $\omega_{0}$ containing $R^{\prime}$ (see Figure 3). Observe that if $R^{\prime}$ can be covered by $N$ sets that are congruent to $R^{\prime} \cap B^{\prime}$ and that have disjoint interiors, then $\widetilde{R^{\prime}}$ is covered by the corresponding sets that are congruent to $\widetilde{R^{\prime}} \cap B^{\prime}$. (This can be proved by the fact that the long side length of $B$ is bigger than that of $R$.) Taking the smallest $N$,

$$
\frac{\left|R^{\prime} \cap B^{\prime}\right|}{\left|\widetilde{R^{\prime}} \cap B^{\prime}\right|}=\frac{N\left|R^{\prime} \cap B^{\prime}\right|}{N\left|\widetilde{R^{\prime}} \cap B^{\prime}\right|} \lesssim \frac{\left|R^{\prime}\right|}{\left|\widetilde{R^{\prime}}\right|} .
$$




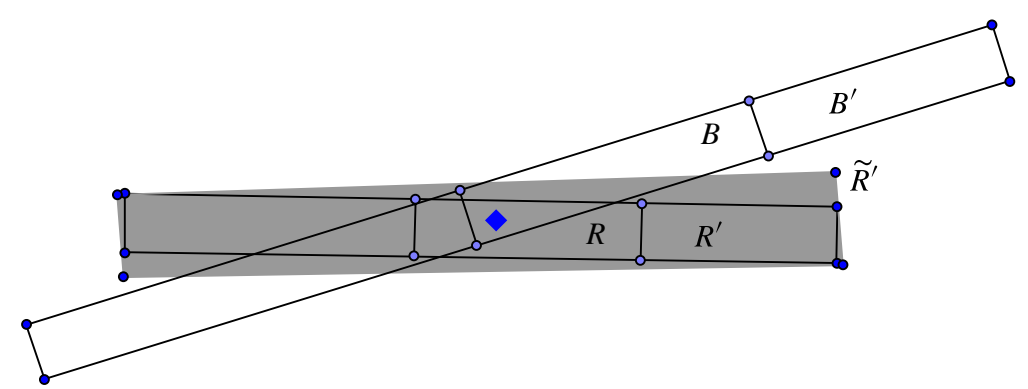

Figure 3. The star shape is the common centre of $R^{\prime}$ and $B^{\prime}$. The rectangle $\widetilde{R^{\prime}}$ is shaded.

We now verify that

$$
\frac{w\left(R^{\prime} \cap B^{\prime}\right)}{w\left(\widetilde{R^{\prime}} \cap B^{\prime}\right)} \lesssim \frac{w\left(R^{\prime}\right)}{w\left(\widetilde{R^{\prime}}\right)} .
$$

Let $P$ be a parallelogram and $P^{\prime}$ be a smallest rectangle containing $P$. Then there exists a rectangle $P^{\prime \prime} \subset P$ such that $P^{\prime}$ is the dilation of $P^{\prime \prime}$ by a factor of eight. From this observation, the doubling property (2.9) and Lemma 2.1, we see that

$$
\begin{aligned}
& w\left(R^{\prime} \cap B^{\prime}\right) \approx \frac{|E|}{\operatorname{rad}(E)} \int_{r_{1}(E)}^{r_{2}(E)} w_{0}(r) d r, \\
& w\left(\widetilde{R^{\prime}} \cap B^{\prime}\right) \approx \frac{|F|}{\operatorname{rad}(F)} \int_{r_{1}(F)}^{r_{2}(F)} w_{0}(r) d r,
\end{aligned}
$$

where $E$ and $F$ are the smallest rectangles containing $R^{\prime} \cap B^{\prime}$ and $\widetilde{R^{\prime}} \cap B^{\prime}$, respectively. By Lemma 2.1 and (2.10), to prove (2.11) we need only verify that

$$
\frac{\frac{1}{\operatorname{rad}(E)} \int_{r_{1}(E)}^{r_{2}(E)} w_{0}(r) d r}{\frac{1}{\operatorname{rad}\left(R^{\prime}\right)} \int_{r_{1}\left(R^{\prime}\right)}^{r_{2}\left(R^{\prime}\right)} w_{0}(r) d r} \lesssim \frac{\frac{1}{\operatorname{rad}(F)} \int_{r_{1}(F)}^{r_{2}(F)} w_{0}(r) d r}{\frac{1}{\operatorname{rad}\left(\widetilde{R^{\prime}}\right)} \int_{r_{1}\left(\widetilde{R^{\prime}}\right)}^{r_{2}\left(\widetilde{R^{\prime}}\right)} w_{0}(r) d r} .
$$

To verify (2.12), we show the following claim.

Claim. There exists a constant $C_{0}>0$ such that

$$
\min \left\{\frac{\operatorname{rad}\left(\widetilde{R^{\prime}}\right)}{\operatorname{rad}\left(R^{\prime}\right)}, \frac{\operatorname{rad}\left(\widetilde{R^{\prime}}\right)}{\operatorname{rad}(F)}\right\} \leq C_{0} .
$$

This claim can be proved by use of Lemma 2.2. If $\widetilde{R^{\prime}}$ contains the origin, then we can easily verify that $\operatorname{rad}\left(\widetilde{R^{\prime}}\right) / \operatorname{rad}\left(R^{\prime}\right) \leq C_{0}$. By symmetry we have only to consider the cases for which $\widetilde{R^{\prime}}$ lies on the upper half plane and $B^{\prime}$ crosses $\widetilde{R^{\prime}}$ from left to right or from bottom to top. For each case we may regard $\widetilde{R^{\prime}} \cap B^{\prime}$ as the segments $\mathrm{B}_{0} \mathrm{~B}_{1}$ or $\mathrm{A}_{0} \mathrm{~B}_{0}$ in Lemma 2.4. Thus, the claim holds.

We return to the proof of Proposition 1.3. 
If $\operatorname{rad}\left(\widetilde{R^{\prime}}\right) / \operatorname{rad}\left(R^{\prime}\right) \leq \operatorname{rad}\left(\widetilde{R^{\prime}}\right) / \operatorname{rad}(F)$ holds, then

$$
r_{2}\left(\widetilde{R^{\prime}}\right)-r_{1}\left(\widetilde{R^{\prime}}\right) \leq C_{0}\left(r_{2}\left(R^{\prime}\right)-r_{1}\left(R^{\prime}\right)\right) .
$$

Hence, using the doubling property of $w_{0}$,

$$
\int_{r_{1}\left(\widetilde{R^{\prime}}\right)}^{r_{2}\left(\widetilde{R^{\prime}}\right)} w_{0}(r) d r \lesssim \int_{r_{1}\left(R^{\prime}\right)}^{r_{2}\left(R^{\prime}\right)} w_{0}(r) d r .
$$

By the supremum condition (1.3) and $E \subset F$,

$$
\begin{aligned}
\frac{1}{\operatorname{rad}(E)} \int_{r_{1}(E)}^{r_{2}(E)} w_{0}(r) d r & \leq \sup _{r_{1}(E)<r<r_{2}(E)} w_{0}(r) \leq \sup _{r_{1}(F)<r<r_{2}(F)} w_{0}(r) \\
& \lesssim \frac{1}{\operatorname{rad}(F)} \int_{r_{1}(F)}^{r_{2}(F)} w_{0}(r) d r .
\end{aligned}
$$

Since $\operatorname{rad}\left(R^{\prime}\right) \leq \operatorname{rad}\left(\widetilde{R^{\prime}}\right)$, we obtain $(2.12)$.

Similarly, if $\operatorname{rad}\left(\widetilde{R^{\prime}}\right) / \operatorname{rad}\left(R^{\prime}\right) \geq \operatorname{rad}\left(\widetilde{R^{\prime}}\right) / \operatorname{rad}(F)$, then

$$
\operatorname{rad}(F) \leq \operatorname{rad}\left(2 \widetilde{R^{\prime}}\right) \lesssim \operatorname{rad}\left(\widetilde{R^{\prime}}\right)
$$

and so, by arguments similar to those above, (2.11) holds.

Finally, let $\widetilde{R}$ be the rectangle with the same centre and whose short side length is three times the length of that of $\widetilde{R^{\prime}}$. Observe that there exists a rectangle $U \subset \mathbb{R}^{2}$ such that $U \subset \widetilde{R} \cap B$ and $\widetilde{R} \cap B^{\prime} \subset \hat{U}$, where $\hat{U}$ is the rectangle expanded from $U$ towards the long sides with lengths five times as big, and hence

$$
w\left(\widetilde{R} \cap B^{\prime}\right) \leq w(\hat{U}) \lesssim w(U) \leq w(\widetilde{R} \cap B) .
$$

Therefore, from $R^{\prime} \subset 6 R, \widetilde{R} \subset 3 \widetilde{R^{\prime}}$ and the doubling property of $w$,

$$
\frac{w(R \cap B)}{w(R)} \lesssim \frac{w\left(R^{\prime} \cap B^{\prime}\right)}{w\left(R^{\prime}\right)} \lesssim \frac{w\left(\widetilde{R^{\prime}} \cap B^{\prime}\right)}{w\left(\widetilde{R^{\prime}}\right)} \lesssim \frac{w\left(\widetilde{R} \cap B^{\prime}\right)}{w(\widetilde{R})} \lesssim \frac{w(\widetilde{R} \cap B)}{w(\widetilde{R})},
$$

where we have used (2.11) in the second inequality. The proof of Proposition 1.3 is now complete.

\section{Proof of Theorem 1.1}

The following argument is due to [2]. We first linearise the operators $M_{\Omega, w}$ and $M_{\Omega_{j}, w}$. For any $\alpha \in \mathbb{Z}^{2}, Q_{\alpha}$ will denote the unit cube centred at $\alpha$. Given a set $\Lambda \subset[0, \pi / 4)$, for each $\alpha$ we choose a rectangle $R_{\alpha} \in \mathcal{B}_{\Lambda}$ such that $R_{\alpha} \supset Q_{\alpha}$. We denote the operator $T_{\Lambda, w}$ by

$$
T_{\Lambda, w} f(x)=\sum_{\alpha} \frac{1}{w\left(R_{\alpha}\right)}\left(\int_{R_{\alpha}} f w\right) \chi_{Q_{\alpha}}(x) .
$$


By definition it is easy to see that

$$
T_{\Lambda, w} f(x) \leq M_{\Lambda, w} f(x)
$$

The following lemma is originally due to Carbery in [3].

Lemma 3.1. Let $T_{\Lambda, w}$ be as above. Then $T_{\Lambda, w}$ is of strong type $(p, p)$ with respect to the measure $w(x) d x$ if and only if there exists a constant $C_{q}$, such that for any sequence $\left\{\lambda_{\alpha}\right\} \subset \mathbb{R}_{+}$,

$$
\int\left(\sum_{\alpha} \lambda_{\alpha} \frac{w\left(Q_{\alpha}\right)}{w\left(R_{\alpha}\right)} \chi_{R_{\alpha}}(x)\right)^{q} w(x) d x \leq C_{q} \sum_{\alpha}\left|\lambda_{\alpha}\right|^{q} w\left(Q_{\alpha}\right),
$$

where $q$ is the conjugate of $p$. Moreover, the infimum of the constants $\left(C_{q}\right)^{1 / q}$ satisfying (3.2) is $\left\|T_{\Lambda}\right\|_{L^{p}(w) \rightarrow L^{p}(w)}$.

Proof. We go through the same argument as for the proof of Theorem 3 in [2].

By Lemma 3.1 with $p=q=2$ it is sufficient to show that inequality (3.2) holds with $C_{2}^{1 / 2}=\sup _{j \geq 1}\left\|M_{\Omega_{j}, w}\right\|_{L^{2}(w) \rightarrow L^{2}(w)}+C\left\|M_{\Omega_{0}, w}\right\|_{L^{2}(w) \rightarrow L^{2}(w)}$.

We write

$$
\begin{aligned}
I^{2}= & \int\left(\sum_{\alpha} \lambda_{\alpha} \frac{w\left(Q_{\alpha}\right)}{w\left(R_{\alpha}\right)} \chi_{R_{\alpha}}(x)\right)^{2} w(x) d x \\
= & \int\left(\sum_{l} \sum_{\alpha: R_{\alpha} \in \Omega_{l}} \lambda_{\alpha} \frac{w\left(Q_{\alpha}\right)}{w\left(R_{\alpha}\right)} \chi_{R_{\alpha}}\right)^{2} w(x) d x \\
= & \int \sum_{l}\left(\sum_{\alpha: R_{\alpha} \in \Omega_{l}} \lambda_{\alpha} \frac{w\left(Q_{\alpha}\right)}{w\left(R_{\alpha}\right)} \chi_{R_{\alpha}}\right)^{2} w \\
& +2 \sum_{l} \sum_{j<l} \int \sum_{R_{\alpha} \in \Omega_{l}} \sum_{R_{\beta} \in \Omega_{j}} \lambda_{\alpha} \lambda_{\beta} \frac{w\left(Q_{\alpha}\right) w\left(Q_{\beta}\right)}{w\left(R_{\alpha}\right) w\left(R_{\beta}\right)} \chi_{R_{\alpha}} \chi_{R_{\beta}} w \\
= & : A+B .
\end{aligned}
$$

For the first term we use (3.1) and Lemma 3.1 with $\Lambda=\Omega_{l}$. We obtain

$$
\begin{aligned}
A & \leq \sum_{l}\left\|M_{\Omega_{l}, w}\right\|_{L^{2}(w) \rightarrow L^{2}(w)}^{2}\left(\sum_{\alpha: R_{\alpha} \in \Omega_{l}}\left|\lambda_{\alpha}\right|^{2} w\left(Q_{\alpha}\right)\right) \\
& \leq\left(\sup _{l}\left\|M_{\Omega_{l}, w}\right\|_{L^{2}(w) \rightarrow L^{2}(w)}^{2}\right)\left(\sum_{l} \sum_{\alpha: R_{\alpha} \in \Omega_{l}}\left|\lambda_{\alpha}\right|^{2} w\left(Q_{\alpha}\right)\right) \\
& \leq\left(\sup _{l}\left\|M_{\Omega_{l}, w}\right\|_{L^{2}(w) \rightarrow L^{2}(w)}^{2}\right)\left(\sum_{\alpha}\left|\lambda_{\alpha}\right|^{2} w\left(Q_{\alpha}\right)\right) .
\end{aligned}
$$

By Proposition 1.3 there exists a constant $C$ such that if $R_{\alpha} \in \Omega_{l}$ and $R_{\beta} \in \Omega_{j}$ with $j<l$, then we can find certain rectangles $\widetilde{R}_{\alpha}^{-}$and $\widetilde{R}_{\beta}^{+}$, containing $R_{\alpha}$ and $R_{\beta}$, respectively, 
pointing in the direction of $\theta_{j}$ and so that

$$
\frac{w\left(R_{\alpha} \cap R_{\beta}\right)}{w\left(R_{\alpha}\right) w\left(R_{\beta}\right)} \lesssim \frac{w\left(\widetilde{R}_{\alpha}^{-} \cap R_{\beta}\right)}{w\left(\widetilde{R}_{\alpha}^{-}\right) w\left(R_{\beta}\right)}+\frac{w\left(R_{\alpha} \cap \widetilde{R}_{\beta}^{+}\right)}{w\left(R_{\alpha}\right) w\left(\widetilde{R}_{\beta}^{+}\right)} .
$$

Observe that both $\widetilde{R}_{\alpha}^{-}$and $\widetilde{R}_{\beta}^{+}$are rectangles of the basis $\mathcal{B}_{0}$. Then

$$
\begin{aligned}
B \leq 2 C & \sum_{l} \sum_{j<l} \int \sum_{R_{\alpha} \in \Omega_{l}} \sum_{R_{\beta} \in \Omega_{j}} \lambda_{\alpha} \lambda_{\beta} \frac{w\left(Q_{\alpha}\right) w\left(Q_{\beta}\right)}{w\left(\widetilde{R}_{\alpha}^{-}\right) w\left(R_{\beta}\right)} \chi_{\widetilde{R}_{\alpha}^{-}} \chi_{R_{\beta}} w(x) \\
& +2 C \sum_{l} \sum_{j<l} \int \sum_{R_{\alpha} \in \Omega_{l}} \sum_{R_{\beta} \in \Omega_{j}} \lambda_{\alpha} \lambda_{\beta} \frac{w\left(Q_{\alpha}\right) w\left(Q_{\beta}\right)}{w\left(R_{\alpha}\right) w\left(\widetilde{R}_{\beta}^{+}\right)} \chi_{R_{\alpha}} \chi_{\widetilde{R}_{\beta}^{+}} w(x) \\
= & B^{-}+B^{+} .
\end{aligned}
$$

We shall only work with $B^{-}$(the other term is analogous). So,

$$
\begin{aligned}
B^{-} & =2 C \sum_{l} \sum_{j<l} \int \sum_{R_{\alpha} \in \Omega_{l}} \sum_{R_{\beta} \in \Omega_{j}} \lambda_{\alpha} \lambda_{\beta} \frac{w\left(Q_{\alpha}\right) w\left(Q_{\beta}\right)}{w\left(\widetilde{R}_{\alpha}^{-}\right) w\left(R_{\beta}\right)} \chi_{\widetilde{R}_{\alpha}^{-}} \chi_{R_{\beta}} w(x) \\
& \leq 2 C \int\left(\sum_{l} \sum_{R_{\alpha} \in \Omega_{l}} \lambda_{\alpha} \frac{w\left(Q_{\alpha}\right)}{w\left(\widetilde{R}_{\alpha}^{-}\right)} \chi_{\widetilde{R}_{\alpha}^{-}} w(x)^{1 / 2}\right)\left(\sum_{j} \sum_{R_{\beta} \in \Omega_{j}} \lambda_{\beta} \frac{w\left(Q_{\beta}\right)}{w\left(R_{\beta}\right)} \chi_{R_{\beta}} w(x)^{1 / 2}\right) .
\end{aligned}
$$

By the Cauchy-Schwarz inequality,

$$
B^{-} \leq 2 C\left(\int\left(\sum_{l} \sum_{R_{\alpha} \in \Omega_{l}} \lambda_{\alpha} \frac{w\left(Q_{\alpha}\right)}{w\left(\widetilde{R}_{\alpha}^{-}\right)} \chi_{\widetilde{R}_{\alpha}^{-}}\right)^{2} w(x)\right)^{1 / 2}\left(\int\left(\sum_{j} \sum_{R_{\beta} \in \Omega_{j}} \lambda_{\beta} \frac{w\left(Q_{\beta}\right)}{w\left(R_{\beta}\right)} \chi_{R_{\beta}}\right)^{2} w(x)\right)^{1 / 2} .
$$

Now notice that $\widetilde{R}_{\alpha}^{-} \in \Omega_{0}$ for all $\alpha$. Then by Lemma 3.1 and (3.1),

$$
B^{-} \leq 2 C\left\|M_{\Omega_{0}, w}\right\|_{L^{2}(w) \rightarrow L^{2}(w)}\left(\sum_{\alpha}\left|\lambda_{\alpha}\right|^{2} w\left(Q_{\alpha}\right)\right)^{1 / 2} I .
$$

Similarly, we can obtain the same bound for $B^{+}$. Combining the bounds (3.3) for $A$ and (3.4) for $B^{ \pm}$,

$$
\begin{gathered}
I^{2} \leq\left(\sup _{l}\left\|M_{\Omega_{l}, w}\right\|_{L^{2}(w) \rightarrow L^{2}(w)}^{2}\right)\left(\sum_{\alpha}\left|\lambda_{\alpha}\right|^{2} w\left(Q_{\alpha}\right)\right) \\
\quad+C\left\|M_{\Omega_{0}, w}\right\|_{L^{2}(w) \rightarrow L^{2}(w)}\left(\sum_{\alpha}\left|\lambda_{\alpha}\right|^{2} w\left(Q_{\alpha}\right)\right)^{1 / 2} I .
\end{gathered}
$$

This implies that

$$
I \leq\left(\sup _{l}\left\|M_{\Omega_{l}, w}\right\|_{L^{2}(w) \rightarrow L^{2}(w)}^{2}+C\left\|M_{\Omega_{0}, w}\right\|_{L^{2}(w) \rightarrow L^{2}(w)}\right)\left(\sum_{\alpha}\left|\lambda_{\alpha}\right|^{2} w\left(Q_{\alpha}\right)\right)^{1 / 2} .
$$

By Lemma 3.1 this completes the proof of Theorem 1.1. 


\section{References}

[1] A. Alfonseca, F. Soria and A. Vargas, 'A remark on maximal operators along directions in $\mathbb{R}^{2}$, Math. Res. Lett. 10(1) (2003), 41-49.

[2] A. Alfonseca, F. Soria and A. Vargas, 'An almost-orthogonality principle in $L^{2}$ for directional maximal functions', in: Harmonic Analysis at Mount Holyoke (South Hadley, MA, 2001), Contemporary Mathematics, 320 (American Mathematical Society, Providence, RI, 2003), 1-7.

[3] A. Carbery, 'Covering lemmas revisited', Proc. Edinburgh Math. Soc. (2) 31(1) (1988), 145-150.

[4] A. Carbery, E. Hernández and F. Soria, 'Estimates for the Kakeya maximal operator on radial functions in $\mathbb{R}^{n}$, in: Harmonic Analysis, ICM-90 Satellite Conference Proceedings (ed. S. Igari) (Springer, Tokyo, 1991), 41-50.

[5] A. Córdoba, 'The Kakeya maximal function and the spherical summation multiplier', Amer. J. Math. 99(1) (1977), 1-22.

[6] J. Duoandikoetxea and V. Naibo, 'The universal maximal operator on special classes of functions', Indiana Univ. Math. J. 54(5) (2005), 1351-1369.

[7] J. García-Cuerva and J. L. Rubio de Francia, Weighted Norm Inequalities and Related Topics (North-Holland, Amsterdam, 1985).

[8] S. Igari, 'On Kakeya's maximal function', Proc. Japan Acad. Ser. A 62(8) (1986), 292-293.

[9] N. H. Katz, 'Maximal operators over arbitrary sets of directions', Duke Math. J. 97(1) (1999), $67-79$.

[10] N. H. Katz, 'Remarks on maximal operators over arbitrary sets of directions', Bull. Lond. Math. Soc. 31(6) (1999), 700-710.

[11] D. S. Kurtz, 'Littlewood-Paley and multiplier theorems on weighted $L^{p}$ spaces', Trans. Amer. Math. Soc. 259(1) (1980), 235-254.

[12] J.-O. Strömberg, 'Maximal functions associated to rectangles with uniformly distributed directions', Ann. of Math. (2) 107(2) (1978), 399-402.

[13] H. Tanaka, 'An elementary proof of an estimate for the Kakeya maximal operator on functions of product type', Tohoku Math. J. (2) 48(3) (1996), 429-435.

[14] H. Tanaka, 'An estimate for the Kakeya maximal operator on functions of square radial type', Tokyo J. Math. 22(2) (1999), 391-398.

HIROKI SAITO, Tokyo Metropolitan University, 1-1 Minami-Ohsawa, Hachioji-shi, Tokyo 192-0397, Japan

e-mail: j1107703@gmail.com

HITOSHI TANAKA, Graduate School of Mathematical Sciences, University of Tokyo, Tokyo 153-8914, Japan

e-mail: htanaka@ms.u-tokyo.ac.jp 